Pricing financial claims contingent upon an underlying asset monitored at discrete times

Green, Ross and Abrahams, I. David and Fusai, Gianluca 2007

MIMS EPrint: 2007.211

Manchester Institute for Mathematical Sciences

School of Mathematics

The University of Manchester

\footnotetext{
Reports available from: http://eprints.maths.manchester.ac.uk/

And by contacting: The MIMS Secretary

School of Mathematics

The University of Manchester

Manchester, M13 9PL, UK
} 


\title{
Pricing financial claims contingent upon an underlying asset monitored at discrete times
}

\author{
Ross Green • I. David Abrahams • \\ Gianluca Fusai
}

Received: 29 June 2007 / Accepted: 11 July 2007 / Published online: 8 August 2007

(C) Springer Science+Business Media B.V. 2007

\begin{abstract}
Exotic option contracts typically specify a contingency upon an underlying asset price monitored at a discrete set of times. Yet, techniques used to price such options routinely assume continuous monitoring leading to often substantial price discrepancies. A brief review of relevant option-pricing methods is presented. The pricing problem is transformed into one of Wiener-Hopf type using a $z$-transform in time and a Fourier transform in the logarithm of asset prices. The Wiener-Hopf technique is used to obtain probabilistic identities for the related random walks killed by an absorbing boundary. An accurate and efficient approximation is obtained using Padé approximants and an approximate inverse $z$-transform based on the trapezoidal rule. For simplicity, European barrier options in a Gaussian Black-Scholes framework are used to exemplify the technique (for which exact analytic expressions are obtained). Extensions to different option contracts and options driven by other Lévy processes are discussed.
\end{abstract}

Keywords Discrete monitoring - Fourier transform - Option pricing · Padé approximants - Wiener-Hopf technique $\cdot z$-transform

\section{Introduction}

\subsection{Conceptual background}

A derivative is an asset whose value is contractually established by the unknown future behaviour of some underlying assets. This work concentrates on pricing derivatives called European barrier options where the underlying asset is monitored discretely. A brief background to option pricing and necessary definitions are given below.

R. Green $(\varangle) \cdot$ I. D. Abrahams

School of Mathematics, University of Manchester,

Manchester M13 9PL, UK

e-mail: rgreen@maths.man.ac.uk

G. Fusai

Dipartimento di Scienze Economiche e Metodi Quantitativi, Universit del Piemonte Orientale Amedeo Avogadro,

Novara, Italy 
A European call option (priced $C_{t}$ at time $t$ ) on an asset (whose price at time $t$ is denoted $S_{t}$ ) gives the holder the right, without obligation, to buy the asset for the strike price $K$ at expiry time $T$. Clearly, ${ }^{1}$ it has payoff $\left(S_{T}-K\right)^{+}$. A European put (priced $P_{t}$ ) gives the holder the right, without obligation, to sell the asset at expiry $T$ for strike price $K$ and has payoff $\left(K-S_{T}\right)^{+}$.

So called 'exotic' contracts, barrier options are like their corresponding 'vanilla' counterparts above, but are 'knocked-out' (become worthless when) or 'knocked-in' (worthless unless) a specified barrier is crossed before expiry. For example, a down-and-out option with barrier level $D$ is rendered worthless if $\underline{S}_{T}=\inf _{0 \leqslant t \leqslant T}\left\{S_{t}\right\}<D$.

\subsection{Mathematical background to option pricing}

\subsubsection{Asset models}

A bank deposit $B_{0}$ is assumed to be risk-free and to accrue interest at rate $r$ so that $B_{t}$ is available for withdrawal at time $t$ with certainty. Seen another way, the risk-free rate of return $r$ is payment for their borrowing capital, compensation for the time value of money. Risk-free investments are modelled by

$\mathrm{d} B_{t}=r B_{t} \mathrm{~d} t, \quad B_{t}=B_{0} \mathrm{e}^{r t}$.

Other investments such as equity stocks $S_{t}$ do not guarantee a return since, for example, trading prices and dividend payments depend on investor sentiment and market conditions. The classical model for risky-assets prices (with drift $\mu$ and volatility $\sigma$ ) is exponential Brownian motion

$\mathrm{d} S_{t}=\mu S_{t} \mathrm{~d} t+\sigma S_{t} \mathrm{~d} W_{t}, \quad S_{t}=S_{0} \mathrm{e}^{X_{t}}$,

where

$\mathrm{d} X_{t}=\left(\mu-\frac{\sigma^{2}}{2}\right) \mathrm{d} t+\sigma \mathrm{d} W_{t}, \quad X_{0}=0$.

It is composed of a deterministic term accounting for expected growth and a random term covering possible variation. Here the random term $W_{t}$ is a Wiener process, ${ }^{2}$ which has stationary, independent, normally distributed increments with mean zero and variance $\Delta t$.

Once underwritten options can subsequently be traded and obtain a market price. When different assets depend on the same source of risk they must be priced consistently, otherwise it may be possible to construct a portfolio which entirely eliminates the risk while guaranteeing a return greater than the risk-free rate. This would give rise to what is known as an arbitrage opportunity (or 'free lunch') and market demand would affect price movements.

Consider a portfolio (value $\Pi_{t}$ ) consisting of a stock $S_{t}$, a European put $P_{t}$ and a short (i.e., the investor has sold or written) call $-C_{t}$ with the same strike $K$ and expiry $T$. At expiry $(t=T)$ the portfolio has value

$\Pi_{T}=S_{T}+\left(K-S_{T}\right)^{+}-\left(S_{T}-K\right)^{+}=K$,

as does a bank deposit of $B_{t}=K \mathrm{e}^{-r(T-t)}$. Clearly, there must be put-call parity

$P_{t}+S_{t}=C_{t}+B_{t}$,

otherwise, without any investment, either borrowing $B_{t}$ and buying the portfolio $\Pi_{t}$ (when $\Pi_{t}<B_{t}$ ) or selling the portfolio $\Pi_{t}$ and lending $B_{t}$ (when $\Pi_{t}>B_{t}$ ) results in a guaranteed profit $\left|\Pi_{t}-B_{t}\right|$.

Similarly, the combination of an up/down in option with an up/down out option clearly replicates the payoff of the corresponding vanilla contract and must be priced accordingly.

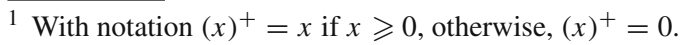

2 A Brownian motion or Wiener process $W_{t}(t \geqslant 0)$ is a continuous trajectory with $W_{0}=0$ whose increments $W_{t}-W_{s}(t \geqslant s)$ are normally distributed with mean 0 and variance $t-s$, and increments for non-overlapping time intervals are independent.
} 
Table 1 Specifics of common option contracts

\begin{tabular}{llll}
\hline Contract & Payoff & Domain & Boundary conditions \\
\hline Long forward & $S_{T}-K$ & {$[0, \infty)$} & \\
European call & $\left(S_{T}-K\right)^{+}$ & {$[0, \infty)$} & 0 as $S_{t} \rightarrow 0, S_{t}$ as $S_{t} \rightarrow \infty$ \\
European put & $\left(K-S_{T}\right)^{+}$ & {$[0, \infty)$} & $K \mathrm{e}^{-r(T-t)}$ as $S_{t} \rightarrow 0,0$ as $S_{t} \rightarrow \infty$ \\
Down and out call & $\left(S_{T}-K\right)^{+}$ & {$[D, \infty)$} & 0 if $S_{t} \leqslant D, S_{t}$ as $S_{t} \rightarrow \infty$ \\
Down and in call & 0 & {$[D, \infty)$} & Call if $S_{t} \leqslant D, 0$ as $S_{t} \rightarrow \infty$ \\
Double barrier & $\left(S_{T}-K\right)^{+}$ & {$[D, U]$} & 0 if $S_{t} \leqslant D$ or $S_{t} \geqslant U$ \\
\hline
\end{tabular}

\subsubsection{PDE approach}

The ideas upon which modern option pricing is based were introduced by Black and Scholes [1]. Modelling an asset price as an exponential Brownian motion (2), they showed that a portfolio obtained by writing an option and buying a quantity (dynamically predetermined by the movement of the call price with respect to the underlying) in the underlying asset,

$V_{t}\left(t, S_{t}\right)=-C_{t}\left(t, S_{t}\right)+\frac{\partial C_{t}}{\partial S_{t}} S_{t}$,

could be constructed which eliminated randomness. The instantaneous (before rebalancing) gain in the portfolio value is

$\mathrm{d} V_{t}=-\mathrm{d} C_{t}+\frac{\partial C_{t}}{\partial S_{t}} \mathrm{~d} S_{t}$,

which by Itô's Lemma ${ }^{3}$ (' $\mathrm{d} W_{t}^{2}=\mathrm{d} t$ ') gives

$\begin{aligned} \mathrm{d} C_{t} & =-\left(\left(\frac{\partial C_{t}}{\partial t}+\mu S_{t} \frac{\partial C_{t}}{\partial S_{t}}+\sigma^{2} S_{t}^{2} \frac{\partial^{2} C_{t}}{\partial S_{t}^{2}}\right) \mathrm{d} t+\sigma S_{t} \frac{\partial C_{t}}{\partial S_{t}} \mathrm{~d} W_{t}\right), \\ \mathrm{d} V_{t} & =-\left(\frac{\partial C_{t}}{\partial t}+\frac{1}{2} \sigma^{2} S_{t}^{2} \frac{\partial^{2} C_{t}}{\partial S_{t}^{2}}\right) \mathrm{d} t .\end{aligned}$

Then, by arbitrage arguments, the growth in this portfolio's value has to be the risk-free rate $r$,

$\mathrm{d} V_{t}=r\left(-C_{t}+\frac{\partial C_{t}}{\partial S_{t}} S_{t}\right) \mathrm{d} t$

from which the famous Black-Scholes PDE is obtained as

$\frac{\partial C_{t}}{\partial t}+r S_{t} \frac{\partial C_{t}}{\partial S_{t}}+\frac{1}{2} \sigma^{2} S_{t}^{2} \frac{\partial^{2} C_{t}}{\partial S_{t}^{2}}=r C_{t}$.

By recourse to a log-transform, Eq. (11) reduces to a (backward) heat equation. Option prices under continuous monitoring are obtained by solving (11) subject to the appropriate boundary and terminal conditions as specified by the payoff function. The specifics for some common option contracts (with strike $K$, lower barrier level $D$ and upper barrier level $U$ ) and the corresponding boundary conditions are compared in Table 1.

Introduce the notation

$\phi(x)=\frac{1}{\sqrt{2 \pi}} \mathrm{e}^{-\frac{x^{2}}{2}}$

${ }^{3}$ The expression ' $\mathrm{d} W_{t}^{2}=\mathrm{d} t$ ' is shorthand for the fact that for any partition $0=t_{0}^{(n)}<t_{1}^{(n)}<\cdots<t_{n}^{(n)}$ of $[0, t]$ with $\max _{j}\left|t_{j+1}^{(n)}-t_{j}^{(n)}\right| \rightarrow$ 0 as $n \rightarrow \infty, \lim _{n \rightarrow \infty} \sum_{j=0}^{n-1}\left|W_{t_{j+1}^{(n)}}-W_{t_{j}^{(n)}}\right|^{2}=t$ in a mean square sense. If $X_{t}=a_{t} \mathrm{~d} t+b_{t} \mathrm{~d} W_{t}\left(a_{t}, b_{t} \in \mathbb{R}\right)$ and $f(x) \in C^{2}(\mathbb{R})$, then Taylor expansion yields $f\left(X_{t}\right)=f\left(X_{0}\right)+\int_{0}^{t} f^{\prime}\left(X_{s}\right) a_{s} \mathrm{~d} s+\int_{0}^{t} f^{\prime}\left(X_{s}\right) b_{s} \mathrm{~d} W_{s}+\frac{1}{2} \int_{0}^{t} f^{\prime \prime}\left(X_{s}\right) b_{s}^{2} \mathrm{~d} s$ or in SDE notation $\mathrm{d} f\left(X_{t}\right)=$ $f^{\prime}\left(X_{t}\right) \mathrm{d} X_{t}+\frac{1}{2} f^{\prime \prime}\left(X_{t}\right) b_{t}^{2} \mathrm{~d} t$. For example, if $Y_{t}=\mathrm{e}^{X_{t}}\left(f(x)=\mathrm{e}^{x}\right)$, then $\mathrm{d} Y_{t}=\left(a_{t}+\frac{1}{2} b_{t}^{2}\right) Y_{t} \mathrm{~d} t+b_{t} Y_{t} \mathrm{~d} W_{t}$. 
for the density of the standard normal distribution and

$\Phi(x)=\int_{-\infty}^{x} \phi(\eta) \mathrm{d} \eta$

for its cumulative value. Then, for the European call option, Eq. 11 has a solution in terms of $\Phi(x)$ :

$C_{t}\left(S_{t}\right)=S_{t} \Phi\left(d_{1}\right)-K \mathrm{e}^{-r(T-t)} \Phi\left(d_{2}\right)$,

where $d_{1}=\frac{\log \left(S_{t} / K\right)+\left(r+\frac{1}{2} \sigma^{2}\right)(T-t)}{\sigma \sqrt{T-t}}$ and $d_{2}=d_{1}-\sigma \sqrt{T-t}$. For the case of the (continuously monitored) downand-out European barrier (level $D$ ) option, Eq. 11 can be solved by the method of images to give

$C_{t}^{\mathrm{Do}}\left(S_{t}\right)=\tilde{C}_{t}\left(S_{t}\right)-\left(\frac{D}{S_{t}}\right)^{\frac{2 r}{\sigma^{2}}-1} \tilde{C}_{t}\left(\frac{D^{2}}{S_{t}}\right)$,

where $\tilde{C}_{t}\left(S_{t}\right)$ is the European call price with payoff $\left(S_{T}-\max \{D, K\}\right)^{+}$. The second term solves (11), equates with $\tilde{C}_{t}\left(S_{t}\right)$ on the boundary $S_{t}=D$ and vanishes on the terminal boundary $(t=T)$ for $S_{T}>\max \{D, K\}$.

\subsubsection{Feynman-Kac formula}

Crucially, in deriving (11) all terms dependent on the drift of the underlying asset are eliminated. Indeed, the drift coefficient $\mu S_{t}$ has been replaced by $r S_{t}$. In probabilistic terms this corresponds to pricing options using a probability measure $\mathbb{Q}$ (equivalent to statistical measure $\mathbb{P}$ ) that makes the discounted underlying asset process $\mathrm{e}^{-r t} S_{t}$ a martingale (or 'fair game' not being expected to win or loose), i.e., $\mathbb{E}\left[\mathrm{e}^{-r T} S_{T} \mid \mathcal{F}_{t}\right]=\mathrm{e}^{-r t} S_{t}$, for all $t$ and $T$ $(t<T)$, where filtration $\mathcal{F}_{t}$ represents the history of the process. Using the new probability measure, the expected return on all financial contracts are equal to the return on the risk-free bank account. In other words, assets are priced using a discounted expectation in a world where investors are indifferent to risk considerations. For this reason the new measure is called the 'risk-neutral probability measure'. Formulation of the fundamentals on the martingale theory of asset pricing are developed in [2-4].

Practically the change from the statistical probability measure $\mathbb{P}$ to the risk-neutral probability measure $\mathbb{Q}$ means instead of using the statistical process

$\mathrm{d} S_{t}=\mu S_{t} \mathrm{~d} t+\sigma S_{t} \mathrm{~d} W_{t}$,

where $W_{t}$ is a $\mathbb{P}$-Brownian motion, the risk-neutral process

$\mathrm{d} S_{t}=r S_{t} \mathrm{~d} t+\sigma S_{t} \mathrm{~d} \tilde{W}_{t}$

is used where $\tilde{W}_{t}=W_{t}+\frac{(\mu-r)}{\sigma} t$ is a $\mathbb{Q}$-Brownian motion (the existence of a suitable $\mathbb{Q}$ is guaranteed by the Cameron-Martin-Girsanov Theorem), so as the discounted price $\tilde{S}_{t}=\mathrm{e}^{-r t} S_{t}$ becomes a martingale, i.e., it is driftless $\mathrm{d} \tilde{S}_{t}=\sigma \tilde{S}_{t} \mathrm{~d} \tilde{W}_{t}$.

The Feynman-Kac Theorem ensures the equivalence between pricing (under no arbitrage) using expectation and solving the Black-Scholes PDE. The Feynman-Kac formula for the price of an European call is

$C_{t}=\mathbb{E}_{\mathbb{Q}}\left[\mathrm{e}^{-r(T-t)}\left(S_{T}-K\right)^{+} \mid \mathcal{F}_{t}\right]$,

and under $\mathbb{Q}$,

$S_{T}=S_{t} \exp \left[\left(r-\frac{\sigma^{2}}{2}\right)(T-t)+\sigma \sqrt{T-t} Z\right]$,

where $Z \sim \mathscr{N}(0,1)$. The solution (14) to the Black-Scholes PDE (11) is obtained as

$$
\begin{aligned}
C_{t} & =\mathrm{e}^{-r(T-t)} \int_{-\infty}^{\infty}\left(S_{t} \mathrm{e}^{\left(r-\frac{\sigma^{2}}{2}\right)(T-t)+\sigma \sqrt{T-t} \xi}-K\right)^{+} \phi(\xi) \mathrm{d} \xi \\
& =S_{t} \int_{-d_{2}}^{\infty} \phi(\xi-\sigma \sqrt{T-t}) \mathrm{d} \xi-K \mathrm{e}^{-r(T-t)} \int_{-d_{2}}^{\infty} \phi(\xi) \mathrm{d} \xi \\
& =S_{t}\left(1-\Phi\left(-d_{2}-\sigma \sqrt{T-t}\right)\right)-K \mathrm{e}^{-r(T-t)}\left(1-\Phi\left(-d_{2}\right)\right) \\
& =S_{t} \Phi\left(d_{1}\right)-K \mathrm{e}^{-r(T-t)} \Phi\left(d_{2}\right) .
\end{aligned}
$$




\subsubsection{Formula in Fourier space}

In this work, the derivation of a pricing formula for (discretely monitored) barrier options is conducted in Fourier space. It is useful to have European call and put prices in the same form. These are now presented.

Denote by $\varphi(x)$ the probability density of $R_{T}=\log \left(S_{T} / S_{t}\right)$ and $w(x)$ an option payoff function in terms of $R_{T}$. Then, employing Parseval's identity, the value of the option is

$V_{t}=\frac{\mathrm{e}^{-r(T-t)}}{2 \pi} \int_{-\infty+\mathrm{i} v}^{\infty+\mathrm{i} v} \hat{w}(-z) \hat{\varphi}(z) \mathrm{d} z$,

where $\hat{w}(z)$ is the Fourier transform of the payoff and $\hat{\varphi}(z)$ is the Fourier transform of the distribution. ${ }^{4}$ The integration path $v=\mathfrak{I m} z$ must lie where both $\hat{w}(z)$ and $\hat{\varphi}(z)$ are well defined. For the (Gaussian) Black-Scholes model, $\hat{\varphi}(z)$ is entire and so allows $v=\mathfrak{I m} z \in(-\infty, \infty)$. For a call,

$\hat{w}(z)=\int_{-\infty}^{\infty} S_{t}\left(\mathrm{e}^{x}-\mathrm{e}^{k}\right)^{+} \mathrm{e}^{\mathrm{i} z x} \mathrm{~d} x=-S_{t} \frac{\mathrm{e}^{(1+\mathrm{i} z) k}}{z(z-\mathrm{i})}, \quad$ Im $z \in(1, \infty)$

and similarly, for a put,

$\hat{w}(z)=\int_{-\infty}^{\infty} S_{t}\left(\mathrm{e}^{x}-\mathrm{e}^{k}\right)^{+} \mathrm{e}^{\mathrm{i} z x} \mathrm{~d} x=-S_{t} \frac{\mathrm{e}^{(1+\mathrm{i} z) k}}{z(z-\mathrm{i})}, \quad \Im \mathfrak{m} z \in(-\infty, 0)$,

where $k=\log \left(K / S_{t}\right)$.

The value of the call is

$C_{t}=-\frac{S_{t} \mathrm{e}^{-r(T-t)}}{2 \pi} \int_{-\infty+\mathrm{i} v}^{\infty+\mathrm{i} v} \mathrm{e}^{(1-\mathrm{i} z) k} \frac{\hat{\varphi}(z)}{z(z+\mathrm{i})} \mathrm{d} z, \quad v>1$.

Normalisation requires $\hat{\varphi}(0)=1$ and the martingale property requires $\hat{\varphi}(-\mathrm{i})=\mathrm{e}^{r(T-t)}$, so the residue of the integrand at its simple poles $z=0$ and $z=-\mathrm{i}$ are $-\frac{K \mathrm{e}^{r(T-t)}}{2 \pi \mathrm{i}}$ and $\frac{S_{t}}{2 \pi \mathrm{i}}$, respectively. Moving the contour to $v=\Im \mathfrak{m} z<0$ picks up the residues to give $C_{t}=P_{t}+S_{t}-K \mathrm{e}^{-r(T-t)}$, where $P_{t}$ is the value of the put, i.e., put-call parity is recovered.

On using partial fractions, the call price can be cast in 'Black-Scholes form',

$$
\begin{aligned}
C_{t} & =\frac{K \mathrm{e}^{-r(T-t)}}{2 \pi \mathrm{i}} \int_{-\infty+\mathrm{i} v}^{\infty+\mathrm{i} v} \mathrm{e}^{-\mathrm{i} k z} \hat{\varphi}(-z)\left(\frac{1}{z-\mathrm{i}}-\frac{1}{z}\right) \mathrm{d} z \\
& =S_{t} I_{1}-K \mathrm{e}^{-r(T-t)} I_{2} .
\end{aligned}
$$

The integrals $I_{1}$ and $I_{2}$ can be interpreted as the option delta $I_{1}=\Delta=\frac{\partial C}{\partial S}$ (since $\frac{\partial I_{1}}{\partial k}=\mathrm{e}^{k} \mathrm{e}^{-r(T-t)} \frac{\partial I_{2}}{\partial k}$ ) and the probability that the option expires in the money $I_{2}=\mathbb{Q}\left(X_{T}>-k\right)=\mathbb{Q}\left(S_{T}>K\right)$.

Even for non-Gaussian models, efficient price calculations are achieved by implementing Fast Fourier Transform, since characteristic functions are generally available in a relatively simple form.

\subsection{Discrete monitoring}

Most traded options do not depend on the entire underlying price path; instead, for practical purposes, contracts specify a discrete set of times at which the underlying price is monitored. Yet, most techniques for pricing discrete options assume continuous monitoring. This allows tractable solutions, but unfortunately the price discrepancies can be large. For knock-out options, continuous time prices are lower than the actual prices, since calculation spuriously disregarded contributions from possible price paths which meander over the barrier between monitoring dates. The price correction amounts to recursive integration with depth equal to the number of monitoring dates. Consequently,

\footnotetext{
${ }^{4}$ The Fourier transform of the probability density $\varphi(x)$ is the characteristic function $\hat{\varphi}(z)=\mathbb{E}_{\mathbb{Q}}\left[\mathrm{e}^{\mathrm{i} z R_{T}} \mid \mathcal{F}_{t}\right]$.
} 
for even relatively few dates, numerical evaluation becomes very inefficient. To overcome the mis-pricing, a wide variety of numerical techniques have been proposed in the literature, including recent noteworthy additions [5-15]. This work is a revision of an article by two of the authors [16] in which an exact analytic expression for the down-out option price was obtained as the solution to the Black-Scholes PDE.

\subsection{Discretely monitored down-and-out barrier options}

Suppose claims are contingent upon $S_{t}=S_{0} \mathrm{e}^{R_{t}}(0 \leqslant t \leqslant T)$ monitored discretely. The log price process $R_{t}(0 \leqslant$ $t \leqslant T$ ) is effectively a random walk with normally distributed increments $\mathscr{N}\left[\left(r-\frac{\sigma^{2}}{2}\right) \Delta t, \sigma^{2} \Delta t\right]$ (under some specified risk-neutral probability measure $\mathbb{Q}$ ) for risk-free rate $r$, volatility $\sigma$ and intermonitoring time $\Delta t$. The transition probability density is

$k(x)=\frac{1}{\sqrt{2 \pi \sigma^{2} \Delta t}} \exp \left[-\frac{\left(x-\left(r-\frac{\sigma^{2}}{2}\right) \Delta t\right)^{2}}{2 \sigma^{2} \Delta t}\right]$.

The payoff of a discrete down-and-out barrier (level $D$ ) European call option with strike $K$ and monitoring times $\mathscr{T}=\{0, \Delta t, \ldots, N \Delta t=T\}$ may be expressed ${ }^{5}$ as

$v\left(R_{N}, \underline{R}_{N}\right)=S_{0}\left(\mathrm{e}^{R_{N}}-\mathrm{e}^{k}\right)^{+} \chi_{[d, \infty)}\left(\underline{R}_{N}\right)$,

where $\underline{R}_{N}=\min _{t \in \mathscr{T}} R_{t}, k=\log K / S_{0}$ and $d=\log D / S_{0}$.

The option price at time $t=0$ is calculated as the discounted expected payoff under the risk-neutral measure $\mathbb{Q}$ given by

$$
\begin{aligned}
C_{D} & =\mathrm{e}^{-r T} \mathbb{E}_{\mathbb{Q}}\left[v\left(R_{N}, \underline{R}_{N}\right)\right] \\
& =\mathrm{e}^{-r T} \int_{-\infty}^{\infty} \int_{0}^{\infty} v(\xi, \zeta) \mathbb{Q}\left(R_{N} \in \mathrm{d} \xi, \underline{R}_{N} \in \mathrm{d} \zeta\right) \\
& =\mathrm{e}^{-r T} \int_{-\infty}^{\infty} w^{(\mathrm{d})}(\xi) \wp_{N}^{(\mathrm{d})}(\xi) \mathrm{d} \xi
\end{aligned}
$$

where $\wp_{N}^{(\mathrm{d})}(\xi)=\mathbb{Q}\left(R_{N} \in \mathrm{d} \xi, \underline{R}_{N-1} \geqslant d\right) / \mathrm{d} \xi$ is the probability density of the random walk $R_{n}$ at time $N$ starting at $R_{0}=0$ and absorbed at boundary $d<0$. The amended payoff is

$$
w^{(\mathrm{d})}\left(R_{N}\right)=S_{0}\left(\mathrm{e}^{R_{N}}-\mathrm{e}^{k}\right)^{+} \chi_{[d, \infty)}\left(R_{N}\right) .
$$

This effectively reduces the pricing problem to evaluating the probabilities $\wp_{N}^{(\mathrm{d})}(\xi)$.

\subsection{Structure of paper}

In the next section the Wiener-Hopf technique is used to obtain analytic expressions for the probability distributions of random walks where paths crossing specified barriers are discarded. Section 3 makes use of these to obtain an exact analytic expression for the price of options as the expected payoff in Fourier space. In Sect. 4 efficient numerical evaluation of prices is achieved by employing Padé approximants. Some concluding remarks and discussions are given in Sect. 5 .

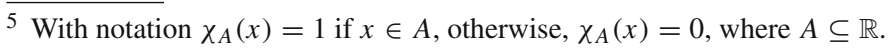




\section{Probability distributions of killed random walks}

Consider a random walk $R_{n}=R_{n-1}+X_{n}, R_{0}=0$, where $X_{n}$ are given independent identically distributed random variables with law $k(x)$. This traces out a path where the transition probability functional that $R_{n+1}=y$ while $R_{n}=x$ is $k(y-x)$.

\subsection{Lower barrier}

Denote by $\wp_{n}^{(\mathrm{d})}(x)$ the probability density that $R_{n}=x$ is realised by a path that does not cross a barrier $d<0$,

$\wp_{n}^{(\mathrm{d})}(x) \mathrm{d} x=\mathbb{P}\left(\left\{R_{n} \in[x, x+\mathrm{d} x)\right\} \cap\left\{\underline{R}_{n-1} \geqslant d\right\}\right)$,

where $\underline{R}_{n}=\min _{0 \leqslant j \leqslant n}\left\{R_{j}\right\}$, then by assumption $X_{n}$ have identical independent distributions,

$\wp_{n}^{(\mathrm{d})}(y)=\int_{d}^{\infty} \wp_{n-1}^{(\mathrm{d})}(x) k(y-x) \mathrm{d} x, \quad \wp_{0}^{(\mathrm{d})}(x)=\delta(x)$.

This Dirac delta function $\delta(x)$ represents a point unit mass at the origin. On taking the z-transform (generating function) of $\wp_{n}^{(\mathrm{d})}(x)$,

$f(x, q)=\mathcal{Z} \wp_{n}^{(\mathrm{d})}(x) \equiv \sum_{n=0}^{\infty} q^{n} \wp_{n}^{(\mathrm{d})}(x), \quad q \in \mathbb{C}$,

it is found that

$f(y, q)-\delta(y)=q \int_{d}^{\infty} f(x, q) k(y-x) \mathrm{d} x$.

The $z$-transform exists within a disk $\mathcal{D}$ which, by Abel's theorem for power series with non-negative coefficients, has radius $\geqslant 1$.

On using a change of variables $(\xi=x-d, v=y-d)$ and defining $g(\xi)=f(\xi+d, q)$ Eq. 39 becomes

$g(v)-\delta(v+d)=q \int_{0}^{\infty} g(\xi) k(v-\xi) \mathrm{d} \xi$.

This is recognisable as a scalar Wiener-Hopf integral equation with kernel $k(v)$; its solution proceeds along the usual lines. A characteristic function may be defined by

$K(z)=\mathcal{F} k(x) \equiv \int_{-\infty}^{\infty} \mathrm{e}^{\mathrm{i} z \xi} k(\xi) \mathrm{d} \xi$

Then using the usual half-range transforms

$G_{-}(z) \equiv \int_{-\infty}^{0} \mathrm{e}^{\mathrm{i} z \xi} g(\xi) \mathrm{d} \xi, \quad G_{+}(z) \equiv \int_{0}^{\infty} \mathrm{e}^{\mathrm{i} z \xi} g(\xi) \mathrm{d} \xi$,

an application of the convolution theorem gives

$G_{+}(z) L(z)=\mathrm{e}^{-\mathrm{i} z d}-G_{-}(z)$,

where

$L(z)=1-q K(z)$.

On the assumption $\mathrm{e}^{-v_{\mp} \xi} g(\xi) \rightarrow 0\left(\nu_{-}<0<v_{+}\right)$as $\xi \rightarrow \pm \infty$, this equation is valid in a strip $\mathcal{S}$ around the origin in which $L(z)$ is analytic and non-zero. On introducing the product factorisations $L(z)=L_{-}(z) L_{+}(z)$ of $L(z)=1-q K(z)$ (into functions which are analytic, zero-free and converge to 1 as $|z| \rightarrow \infty$ below/above this strip, respectively) and the sum factorisation $P(z)=P_{-}(z)+P_{+}(z)$ of

$P(z)=\mathrm{e}^{-\mathrm{id} z} / L_{-}(z)$ 
(into functions which are analytic and converge to 0 as $|z| \rightarrow \infty$ below/above this strip), Eq. 43 may be expressed as

$G_{+}(z) L_{+}(z)-P_{+}(z)=P_{-}(z)-G_{-}(z) / L_{-}(z)$.

This equates (within strip $\mathcal{S}$ ) a function analytic in and above $\mathcal{S}$ to a function analytic in and below $\mathcal{S}$, and so, by analytic continuation, defines an entire function.

On the assumption $g(\xi)=f(\xi+d)$ is bounded at $\xi= \pm 0, G_{ \pm}(z) \sim 1 / z$ as $|z| \rightarrow \infty(\mathfrak{I m} z \gtrless 0)$, so by Liouville's theorem (46) equates to zero, giving

$G_{+}(z)=\frac{P_{+}(z)}{L_{+}(z)}=\frac{\mathrm{e}^{-\mathrm{i} d z}}{L(z)}-\frac{P_{-}(z) L_{-}(z)}{L(z)}$.

Explicit integral expressions for these factorisations are

$P_{ \pm}(z)= \pm \frac{1}{2 \pi i} \int_{-\infty}^{\infty} \frac{\mathrm{e}^{-\mathrm{id} \zeta}}{L_{-}(\zeta)(\zeta-z)} \mathrm{d} \zeta, \quad \mathfrak{I m} \zeta \lessgtr \mathfrak{I m} z$

and

$L_{ \pm}(z)=\exp \left\{ \pm \frac{1}{2 \pi \mathrm{i}} \int_{-\infty}^{\infty} \frac{\log L(\zeta)}{\zeta-z} \mathrm{~d} z\right\}, \quad \mathfrak{I m} \zeta \lessgtr \mathfrak{I m} z$

The probabilities $\wp_{n}^{(\mathrm{d})}(x)$ are recovered as

$$
\begin{aligned}
\wp_{n}^{(\mathrm{d})}(x) & =\mathcal{Z}_{n}^{-1} g(x-d) \\
& = \begin{cases}\mathcal{Z}_{n}^{-1} \mathcal{F}^{-1} \mathrm{e}^{\mathrm{id} z} G_{+}(z) & \text { for } x>d, \\
\mathcal{Z}_{n}^{-1} \mathcal{F}^{-1} \mathrm{e}^{\mathrm{id} z} G_{-}(z) & \text { for } x<d .\end{cases}
\end{aligned}
$$

On substituting (47) in (51) and taking the inverse transform, it is found, for $x>d$, that

$$
\begin{aligned}
\wp_{n}^{(\mathrm{d})}(x) & =\mathcal{Z}_{n}^{-1} \mathcal{F}^{-1}\left(\frac{1}{L(z)}-\mathrm{e}^{\mathrm{id} z} \frac{P_{-}(z) L_{-}(z)}{L(z)}\right) \\
& =\wp_{n}(x)-\frac{\mathcal{Z}_{n}^{-1}}{2 \pi}\left(\int_{-\infty}^{\infty} \frac{P_{-}(z) L_{-}(z)}{L(z)} \mathrm{e}^{-\mathrm{i}(x-d) z} \mathrm{~d} z\right),
\end{aligned}
$$

where $\wp_{n}(x)$ is the probability density without barriers.

\subsubsection{Inverse Fourier transform}

The path of the inverse Fourier transform should lie within the analytic strip $\mathcal{S}$. Integrals may be evaluated numerically, but their convergence is slow. Although convergence is greatly improved by splitting the integrals into two using $1 / L(z)=q K(z) / L(z)+1$, calculations remain too slow for practical purposes.

\subsubsection{Inverse z-transform}

The inverse $z$-transform is the Taylor coefficient of $q^{N}$, which is given by

$\mathcal{Z}_{N}^{-1} f(q)=\frac{1}{2 \pi \mathrm{i}} \oint_{\Lambda} f(q) q^{-(N+1)} \mathrm{d} q$

where $\Lambda \in \mathcal{D}$ is a contour enclosing $q=0$. Choosing a circular (radius $\rho<1$ ) integration contour, the inverse $z$-transform (54) expressed in polar coordinates is

$\mathcal{Z}_{N}^{-1} f(q)=\frac{1}{2 \pi \rho^{N}} \int_{0}^{2 \pi} f\left(\rho \mathrm{e}^{\mathrm{i} \theta}\right) \mathrm{e}^{-\mathrm{i} N \theta} \mathrm{d} \theta$.

This may be evaluated by numerical integration, but for large $N$ this becomes extremely slow. 
Abate and Whitt [17] propose an alternative method based on approximating (55) using the trapezoidal rule with step size $\pi / N$,

$\mathcal{Z}_{N}^{-1} f(q) \approx \frac{1}{2 \pi \rho^{N}}\left\{f(\rho)+(-1)^{N} f(-\rho)+2 \sum_{j=1}^{N-1}(-1)^{j} \mathfrak{R e}\left[f\left(\rho \mathrm{e}^{j \pi \mathrm{i} / N}\right)\right]\right\}$.

This has an error bound $\rho^{2 N} /\left(1-\rho^{2 N}\right)$, so practically, to have an accuracy of at least $10^{-\alpha}$ requires $\rho=10^{-\alpha / 2 N}$. It is important to adjust $\rho$ in this way so calculation of $\rho^{N}=10^{-\alpha / 2}$ in the denominator of (56) does not introduce error.

\subsection{Upper barrier}

The above analysis can be repeated in the case of an upper barrier. Denote by $\wp_{n}^{(\mathrm{u})}(x)$ the probability density that $R_{n}=x$ is realised by a path that does not cross a barrier $u>0$,

$\wp_{n}^{(\mathrm{u})}(x) d x=\mathbb{P}\left(\left\{R_{n} \in[x, x+d x)\right\} \cap\left\{\bar{R}_{n-1} \leqslant u\right\}\right)$,

where $\bar{R}_{n}=\sup _{0 \leqslant j \leqslant n}\left\{R_{j}\right\}$, then

$\wp_{n}^{(\mathrm{u})}(x)=\wp_{n}(x)-\frac{\mathcal{Z}_{n}^{-1}}{2 \pi}\left(\int_{-\infty}^{\infty} \frac{Q_{+}(z) L_{+}(z)}{L(z)} \mathrm{e}^{-\mathrm{i}(x-u) z} \mathrm{~d} z\right)$

where

$Q_{+}(z)=\frac{1}{2 \pi \mathrm{i}} \int_{-\infty}^{\infty} \frac{\mathrm{e}^{-\mathrm{i} u \zeta}}{L_{+}(\zeta)(\zeta-z)} \mathrm{d} \zeta, \mathfrak{I m} \zeta<\mathfrak{I m} z$.

\section{Exact expressions for barrier options}

\subsection{Discretely monitored down-and-out barrier options}

On resuming the calculation of the down-and-out call option form (34), taking Fourier and $z$-transforms, employing Parseval's relation and recognising the random walk result (51) it is found that

$$
\begin{aligned}
C_{D} & =\frac{\mathrm{e}^{-r T} \mathcal{Z}_{N}^{-1}}{2 \pi} \int_{-\infty+\mathrm{i} v}^{\infty+\mathrm{i} v} \mathcal{F} w^{(\mathrm{d})}(\xi)^{*} \mathcal{Z} \mathcal{F} \wp_{N}^{(\mathrm{d})}(\xi) \mathrm{d} \xi \\
& =\frac{\mathrm{e}^{-r T} \mathcal{Z}_{N}^{-1}}{2 \pi} \int_{-\infty+\mathrm{i} v}^{\infty+\mathrm{i} v} \hat{w}^{(\mathrm{d})}(-z) G_{+}(z) \mathrm{e}^{\mathrm{id} z} \mathrm{~d} z \\
& =\frac{\mathrm{e}^{-r T} \mathcal{Z}_{N}^{-1}}{2 \pi} \int_{-\infty+\mathrm{i} v}^{\infty+\mathrm{i} v} \hat{w}^{(\mathrm{d})}(-z)\left(\frac{1}{L(z)}-\mathrm{e}^{\mathrm{id} z} \frac{P_{-}(z) L_{-}(z)}{L(z)}\right) \mathrm{d} z \\
& =\tilde{C}_{D}-\frac{\mathrm{e}^{-r T} \mathcal{Z}_{N}^{-1}}{2 \pi} \int_{-\infty+\mathrm{i} v}^{\infty+\mathrm{i} v} \hat{w}^{(\mathrm{d})}(-z)\left(\mathrm{e}^{\mathrm{id} z} \frac{P_{-}(z) L_{-}(z)}{L(z)}\right) \mathrm{d} z,
\end{aligned}
$$

where $\tilde{C}_{D}$ is the vanilla option price with payoff $w^{(\mathrm{d})}\left(R_{N}\right)$ (exactly analogous to the continuous time-option-pricing formula).

The transformed payoff,

$$
\begin{aligned}
\hat{w}^{(\mathrm{d})}(z) & =\int_{d}^{\infty} S_{0}\left(\mathrm{e}^{x}-\mathrm{e}^{k}\right)^{+} \mathrm{e}^{\mathrm{i} z x} \mathrm{~d} x \\
& =-S_{0}\left[\frac{\mathrm{e}^{(1+i z) \max (d, k)}}{1+\mathrm{i} z}-\frac{\mathrm{e}^{k+\mathrm{i} z \max (d, k)}}{\mathrm{i} z}\right],
\end{aligned}
$$

is valid for $\mathfrak{I m} z>1$. 
The characteristic function (Fourier transform of (30)) $K(z)$ for the log-price increments is

$K(z)=\exp \left\{\mathrm{i} z\left(r-\sigma^{2} \Delta t / 2\right)-z^{2} \sigma^{2} \Delta t / 2\right\}$,

valid for all $z$. An arbitrarily wide strip about the origin can be made free of the zeros of $L(z)=1-q K(z)$ by making $|q|$ sufficiently small. In such circumstances the above integral is defined for any contour in the analytic $\operatorname{strip} \mathcal{S}$ with $v=\mathfrak{I m} z<-1$.

\subsubsection{Exact sum solution by residue calculus}

The second term in the integrand of (63) is analytic in the lower-half plane ( $\mathfrak{I m} z<-1)$ except at the zeros of $L(z)$ and vanishes as $|z| \rightarrow \infty$. On deforming the integration contour downwards $C_{D}$ may be expressed as

$C_{D}=\tilde{C}_{D}+\mathrm{ie}^{-r T} \mathcal{Z}_{N}^{-1} \sum_{n=-\infty}^{\infty}\left(P_{-}\left(\gamma_{n}^{-}\right) L_{-}\left(\gamma_{n}^{-}\right) R\left(\gamma_{n}^{-}\right) \hat{w}^{(\mathrm{d})}\left(-\gamma_{n}^{-}\right) \mathrm{e}^{\mathrm{id} \gamma_{n}^{-}}\right)$,

where the summation is over all the residues arising from poles $\gamma_{n}^{-}$of $1 / L(z)$ in $\mathfrak{I m}(z)<0, R\left(\gamma_{n}^{-}\right)$. Similarly,

$P_{-}(z)=\sum_{n=-\infty}^{\infty} \frac{\mathrm{e}^{-\mathrm{id} \gamma_{n}^{+}} L_{+}\left(\gamma_{n}^{+}\right) R\left(\gamma_{n}^{+}\right)}{\gamma_{n}^{+}-z}$.

It remains for $L_{ \pm}\left(\gamma_{n}^{ \pm}\right)$to be approximated or evaluated by numerical integration.

When $d \ll 0$ (i.e., $D \ll S_{0}$ ), these sums converge rapidly due to the exponential terms. Yet, as $d \rightarrow 0$ their convergence becomes slow and, to maintain accuracy, many terms are required making the numerical evaluation inefficient.

The solutions of $L\left(\gamma_{n}\right)=1-q K\left(\gamma_{n}\right)=0$ are given by

$\gamma_{n}^{ \pm}=\frac{\mathrm{i}\left(r-\sigma^{2} \Delta t / 2\right)}{\sigma^{2} \Delta t} \pm \frac{1}{\sigma \sqrt{\Delta t}} \sqrt{2 \log q+4 n \pi \mathrm{i}-\frac{\left(r-\sigma^{2} \Delta t / 2\right)^{2}}{\sigma^{2} \Delta t}}(n \in \mathbb{Z})$.

The residue $R(\gamma)$ at the simple pole $\gamma$ of $1 / L(z)$ is calculated as

$R(\gamma)=\frac{1}{\gamma \sigma^{2} \Delta t-\mathrm{i}\left(r-\sigma^{2} \Delta t / 2\right)}$.

\subsection{Discretely monitored up-and-out barrier options}

The value of a discrete up-and-out barrier (level $U$ ) call option with strike $K$ and monitoring times $\mathscr{T}=\{0, \Delta t, \ldots$, $N \Delta t=T\}$ is

$C_{U}=\tilde{C}_{U}-\frac{\mathcal{Z}_{N}^{-1}}{2 \pi} \int_{-\infty+\mathrm{i} v}^{\infty+\mathrm{i} v} \hat{w}^{(\mathrm{u})}(-z)\left(\mathrm{e}^{\mathrm{i} u z} \frac{Q_{+}(z) L_{+}(z)}{L(z)}\right) \mathrm{d} z$,

where

$\hat{w}^{(u)}(z)=S_{0}\left[\frac{\mathrm{e}^{(1+\mathrm{i} z) u}}{1+\mathrm{i} z}-\frac{\mathrm{e}^{k+\mathrm{i} z u}}{\mathrm{i} z}\right]-S_{0}\left[\frac{\mathrm{e}^{(1+\mathrm{i} z) k}}{1+\mathrm{i} z}-\frac{\mathrm{e}^{k+\mathrm{i} z k}}{\mathrm{i} z}\right]$

is valid in $\mathfrak{I m} z<0$, and $u=\log U / S_{0}>0$. The integration path must lie within the analytic strip with $v=\mathfrak{I m} z>0$. The price of the corresponding vanilla option with payoff $\hat{w}^{(\mathrm{u})}(z)$ is denoted $\tilde{C}_{U}$.

For the down-and-out option, the barrier had no effect on the transformed amended payoff when below the strike. Yet, the up-and-out barrier always has influence. This manifests itself in slow convergence even for options where $u \gg 0$. Consequently, the product factorisation $L_{ \pm}(z)$ is best accomplished via a fast approximate approach rather than via the Cauchy integral representation given in (49). Thus, a Padé approximant approach is far more efficient in evaluating (71). 


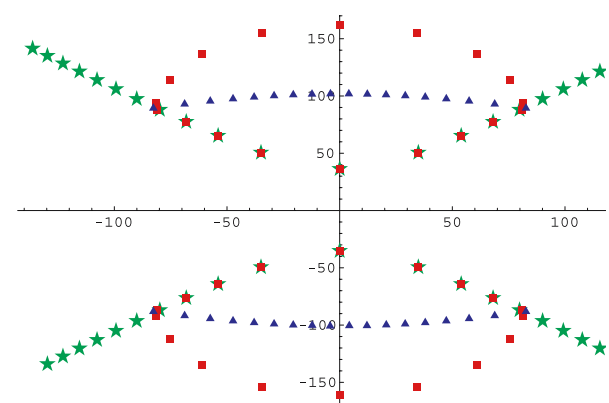

Fig. 1 Example of smallest exact zeros $(\star)$ and plot of zeros $(\square)$ and poles $(\boldsymbol{\Lambda})$ of an approximation to $L(z)=1-q K(z)$ with [24/36] Padé approximant to $K(z)$

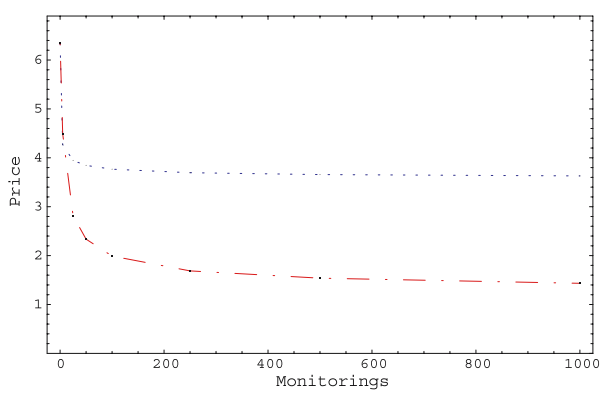

Fig. 2 Example behaviour of discrete up-out $(\cdots)$ and down-out $(\cdot-\cdot)$ barrier prices as monitoring frequency increases

\section{Approximate prices using Padé approximants}

Option prices are calculated most efficiently by obtaining an [L/M] Padé rational approximation of $K(z)$, which is the quotient of a polynomial of degree $L$ to one of degree $M$. Once calculated, the factorisation of $L(z)=1-q K(z)$ into non-zero analytic functions in $\mathfrak{I m} z \gtrless 0$ follows by inspection after polynomial factorisation. For details on the approximation of Wiener-Hopf kernels by Padé approximants the reader is referred to the article by Abrahams [18]. Evaluation of $P_{-}(z)$ and $Q_{+}(z)$, and in turn (63) and (71), comprise only the summation of a small number of residues calculated algebraically.

On using a [24/36] Padé approximant for $K(z)$, results are achieved to an accuracy of at least five decimal places. This accuracy is relatively insensitive to the choice of Padé number, but this particular choice gives rapid decay for large $|z|$. Computational time in Mathematica is an order of a few seconds for hundreds of monitorings and increases at most linearly with monitoring frequency. For example options with $S_{0}=K=100, r=0.1, \sigma=0.3$ and $T=0.2$, Fig. 1 contrasts the zero-pole plot of the Padé approximant with the exact zeros of $L(z)$ and Fig. 2 demonstrates the convergence of the down-out option with barrier level $D=99$ and up-out option with barrier level $U=125$ to the continuously monitored prices as the monitoring frequency is increased. Asymptotic analysis confirms the difference in continuous and discrete prices is proportional to $\sqrt{\Delta t}$ as frequency increases. Economically this means even with relatively frequent monitoring the difference between the real price and that calculated assuming continuous monitoring can remain significant. The price calculated for the down-out option with a million monitorings (approximately ever second of the working day) is 1.1794 , which compares to the continuous price 1.1708 .

\section{Discussion and conclusion}

A combined z-transform, Fourier transform and Wiener-Hopf-type argument has provided a novel technique to price discrete barrier options. Accurate approximations are achieved by expressing the solution entirely in terms of Padé approximants and approximating the inverse $z$-transform using a method based on the trapezoidal rule. A (Gaussian) Black-Scholes example demonstrated that the mis-pricing incurred by a continuous monitoring approximation can be substantial even for relatively frequent monitoring.

The extension of this work to a variety of other options has been completed. For example, double-barrier pricing demands a modification of the Wiener-Hopf technique, observing a simple probabilistic relationship gives the hindsight price and the analytic continuation of the barrier results presented here essentially give first touch prices. In the frequent monitoring limit the formulae obtained yield asymptotic results related to the continuity correction introduced by Broadie et al. [7]. 
The extension of this work to a non-Gaussian framework (with underlying assets driven by different Lévy processes which more accurately model market data) involves only the substitution of a different characteristic function. For some processes accurate Padé approximation presents some technical difficulties. The characteristic functions of many Lévy processes have complicated structures, including branch cuts, making them inconvenient to use and calculations cumbersome. Here Padé approximants are used to simplify the characteristic function and subsequent calculation. The feasibility of modelling with a rational characteristic function (instead of adopting processes ad hoc) is an interesting line of present enquiry by the authors.

\section{References}

1. Black F, Scholes M (1973) The pricing of options and corporate liabilities. J Polit Economy 81:637-654

2. Harrison JM, Kreps DM (1979) Martingales and arbitrage in multiperiod securities markets. J Econ Theory 20:381-408

3. Harrison JM, Pliska SR (1981) Martingales and stochastic integrals in the theory of continuous trading. Stochastic Process Appl 11:215-260

4. Delbaen F, Schachermayer W (1994) A general version of the fundamental theorem of asset pricing. Math Ann 300:463-520

5. Airoldi M (2005) A moment expansion approach to option pricing. Quant Finance 5(1):89-104

6. Borovkov K, Novikov A (2002) On a new approach to calculating expectations for option pricing. J Appl Probab 39(4):889-895

7. Broadie M, Glasserman P, Kou S (1997) A continuity correction for discrete barrier options. Math Finance 7:325-349

8. Broadie M, Glasserman P, Kou S (1999) Connecting discrete and continuous path-dependent options. Finance Stoch 3:55-82

9. Broadie M, Yamamoto Y (2005) A double-exponential fast Gauss transform algorithm for pricing discrete path-dependent options. Oper Res 53(5):764-779

10. Fusai G, Recchioni MC (2007) Analysis of quadrature methods for pricing discrete barrier options. J Econom Dynam Control 31:826-860

11. Hörfelt P (2003) Extension of the corrected barrier approximation by Broadie, Glasserman, and Kou. Finance Stoch 7:231-243

12. Howison S, Steinberg M (2007) A matched asymptotic expansion approach to continuity corrections for discretely sampled options. Part 1: barrier options-Appl Math Finance 14(1):63-89

13. Kou SG (2003) On pricing of discrete barrier options. Statist Sinica 13:955-964

14. Petrella G, Kou S (2004) Numerical pricing of discrete barrier and lookback options via Laplace transforms. J Comp Finance $8(1): 1-38$

15. Tse WM, Li LK, Ng KW (2001) Pricing discrete barrier and hindsight options with the tridiagonal probability algorithm. Manage Sci 47(3):383-393

16. Fusai G, Abrahams ID, Sgarra C (2006) An exact analytical solution for discrete barrier options. Finance Stoch 10(1):1-26

17. Abate J, Whitt W (1992) Numerical inversion of probability generating functions. Oper Res Lett 12:245-251

18. Abrahams ID (2000) The application of Padé approximants to Wiener-Hopf factorization. IMA J Appl Math 65:257-281 